\title{
The Transition Metal-Catalyzed Addition of C-H Bonds in Aromatic Hydrazones to Olefins
}

\author{
Fumitoshi Kakiuchi, Takuya Tsujimoto, Motohiro Sonoda, Naoto Chatani, Shinji Murai* \\ Department of Applied Chemistry, Faculty of Engineering, Osaka University, Suita, Osaka 565-0971, Japan \\ Fax +81 6 68797396; E-mail: murai@ chem.eng.osaka-u.ac.jp \\ Received 18 January 2001
}

\begin{abstract}
Catalytic additions of C-H bonds in aromatic hydrazones to olefins proceeded with the aid of ruthenium or rhodium complexes. Several hydrazones can be used in the chelation-assisted C-H/ olefin coupling described herein. When a $\mathrm{Ru}_{3}(\mathrm{CO})_{12}$ complex was used as the catalyst, a mixture of the 1:1 addition product, along with the corresponding dehydrogenated coupling products was obtained. In the case of the reaction using $\mathrm{RhCl}\left(\mathrm{PPh}_{3}\right)_{3}$-catalyst, no dehydrogenated coupling product was obtained and the 1:2 addition product was produced as the major product.
\end{abstract}

Key words: ruthenium catalysts, rhodium catalysts, C-H bond cleavage, aromatic hydrazones, $\mathrm{C}$-H/olefin coupling

As the result of recent progress in the area, catalytic reactions involving $\mathrm{C}-\mathrm{H}$ bond cleavage has been found to be one of the most useful protocols for the C-C bond formation. ${ }^{1} \mathrm{We}$ have been investigating the transition metal-catalyzed addition of $\mathrm{C}-\mathrm{H}$ bonds to $\mathrm{C}-\mathrm{C}$ multiple bonds in which chelation-assistance by directing groups is believed to play a major role in the catalytic cycle, especially in the C-H bond cleavage step. ${ }^{1-12}$ To date, we have demonstrated that various types of aromatic and olefinic compounds having ketone ${ }^{2}{ }^{\text {ester }}{ }^{4}$ imine,${ }^{6}$ imidate, ${ }^{7}$ nitrile,${ }^{8}$ aldehyde, ${ }^{9}$ and pyridyl moieties ${ }^{10}$ can be applied to chelationassisted $\mathrm{C}-\mathrm{H} /$ olefin coupling. In this communication, we wish to report that an $s p^{2}$ nitrogen in hydrazones, which are often used in organic synthesis for the preparation of nitriles ${ }^{13}$ and chiral amines, ${ }^{14}$ can also be used as a directing group for $\mathrm{C}-\mathrm{H} /$ olefin coupling, giving the corresponding ortho alkylated products.

The reaction of hydrzone $\mathbf{1}$, derived from acetophenone, with triethoxyvinylsilane (2) was carried out using $\mathrm{Ru}_{3}(\mathrm{CO})_{12}$ (3) as a catalyst (eq 1$) .{ }^{15}$ The $1: 1$ addition product 4 and the corresponding dehydrogenative coupling products 5 were obtained in $46 \%$ and $41 \%$ yields, respectively. ${ }^{16,17}$

The catalytic activities of the $\mathrm{RuH}_{2}(\mathrm{CO})\left(\mathrm{PPh}_{3}\right)_{3}$ and $\mathrm{RhCl}\left(\mathrm{PPh}_{3}\right)_{3}$ complexes were also examined, and the results are shown in Table 1 . When the ruthenium-phosphine complex, $\mathrm{RuH}_{2}(\mathrm{CO})\left(\mathrm{PPh}_{3}\right)_{3}$, was used instead of complex 3, 1 was completely consumed and $\mathbf{4}$ and $\mathbf{5}$ were obtained in $89 \%$ and $11 \%$ yields, respectively, with no 1:2 addition product (run 2). In contrast to the case of the reaction of aromatic ketimine $\mathbf{8}$ with $\mathbf{2}$ which gave no dehydrogenated products, ${ }^{6 a}$ the reaction of the ketone hydrazone 1 afforded a considerable amount of dehydrogenated product 5 (runs 1 and 2). When Wilkinson's cat-

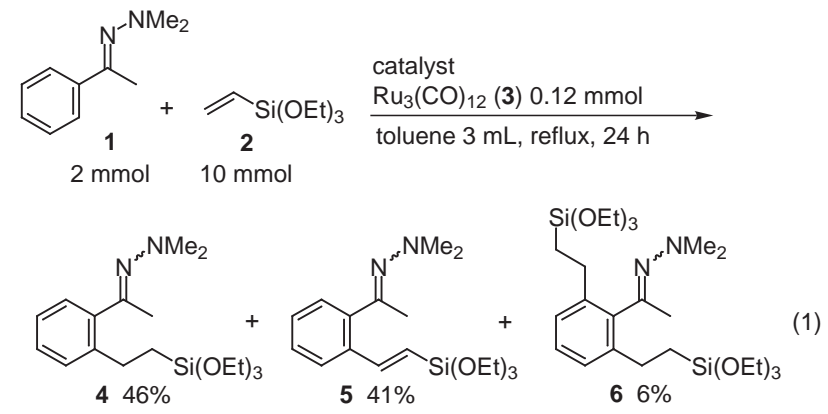

Scheme 1

alyst (7) was used, the 1:2 addition product 6 was obtained as a major product, after prolonged reaction period (48 $\mathrm{h}$ ) (run 3). Although the $\mathrm{Ru}_{3}(\mathrm{CO})_{12}$-catalyzed reaction of the aromatic ketimine $\mathbf{8}$ with $\mathbf{2}$ gave only the corresponding $1: 1$ addition product, ${ }^{6 a}$ the use of catalyst 7 for the reaction of $\mathbf{1}$ with $\mathbf{2}$ resulted in the 1:2 addition product $\mathbf{6}$ as a major product (runs 3-5). Under forcing reaction conditions, i.e., using refluxing mesitylene as the solvent, a high total yield of coupling products 4 and $\mathbf{6}$ (98\% combined yield) was achieved. Even when a smaller amount of the olefin 2 was used (run 5), the 1:2 coupling product was obtained as a major product $(\mathbf{4}, 34 \%$ yield and $\mathbf{6}, 55 \%$ yield). The predominant formation of the 1:2 addition product 6 can be attributed to the binding affinity of the nitrogen atom for the rhodium center, in which the second $\mathrm{C}-\mathrm{H}$ bond cleavage leading to the formation of 1:2 addition product took place without the dissociation of the $1: 1$ product from the rhodium center. ${ }^{18}$

Table 1 The Reaction of Ketone Hydrazone 1 with Triethoxyvinylsilane (2) Using a Ruthenium or Rhodium Catalyst ${ }^{\mathrm{a}}$

\begin{tabular}{lllrrrr}
\hline \multirow{2}{*}{ run } & \multirow{2}{*}{ catalyst } & \multirow{2}{*}{ solvent } & time & \multicolumn{4}{c}{ yields, \% } \\
\cline { 5 - 8 } & & & & $\mathbf{4}$ & $\mathbf{5}$ & $\mathbf{6}$ \\
\hline 1 & $\mathrm{Ru}_{3}(\mathrm{CO})_{12}(\mathbf{3})$ & toluene & $24 \mathrm{~h}$ & 46 & 41 & 6 \\
2 & $\mathrm{RuH}_{2}(\mathrm{CO})\left(\mathrm{PPh}_{3}\right)_{3}$ & toluene & $48 \mathrm{~h}$ & 89 & 11 & 0 \\
3 & $\mathrm{RhCl}_{\left(\mathrm{PPh}_{3}\right)_{3}(\mathbf{7})}$ & toluene & $48 \mathrm{~h}$ & 16 & 0 & 75 \\
4 & $\mathrm{RhCl}_{\left(\mathrm{PPh}_{3}\right)_{3}}$ & mesitylene & $6 \mathrm{~h}$ & 27 & 0 & 71 \\
$5^{\mathrm{b}}$ & $\mathrm{RhCl}\left(\mathrm{PPh}_{3}\right)_{3}$ & mesitylene & $24 \mathrm{~h}$ & 34 & 0 & 55 \\
\hline
\end{tabular}

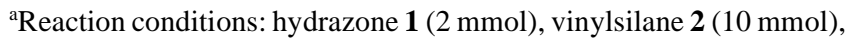
catalyst $(0.12 \mathrm{mmol})$, solvent $3 \mathrm{~mL}$, reflux.

${ }^{\mathrm{b}}$ Two equivalents of $\mathbf{2}$ was used. 
<smiles>CC(=NBr)c1ccccc1</smiles>

Figure 1

The reaction of several hydrazones with olefins was examined, and some selected results are listed in Table 2. The ketone hydrazone 9 gave the 1:1 (10) and 1:2 addition products (11) in $27 \%$ and $73 \%$ yields, respectively (Table 2 , run 1 ). In this case, the reactivity of the hydrazone was very similar to the $N, N$-dimethylamino derivative 1 . The reaction of hydrazone $\mathbf{1}$ with ethylene gave the ortho ethylated products $\mathbf{1 2}$ and $\mathbf{1 3}$ in 5\% and 88\% yields, respectively (Table 2, run 2). The reaction with $o$-methylstyrene proceeded smoothly to give only the 1:1 addition product 14 (Table 2, run 3). In the course of our studies of C-H/ olefin coupling, the use of aromatic olefins such as styrenes usually resulted in the exclusive formation of the corresponding 1:1 addition product. The reaction of hydrazone 15, derived from $o$-methylbenzaldehyde, with vinylsilane 2 was carried using the $\mathrm{Ru}_{3}(\mathrm{CO})_{12}$-catalyst. The 1:1 addition product $\mathbf{1 6}$ and the dehydrogenated product 17 were obtained in $30 \%$ and $8 \%$ yields, respectively (Table 2 , run 4). The catalytic activities of several other ruthenium complexes were also examined. Of the catalysts screened, $\mathrm{RuH}_{2}(\mathrm{CO})\left(\mathrm{PPh}_{3}\right)_{3}(\mathbf{1 6}, 13 \% ; \mathbf{1 7}, 15 \% ; 48 \mathrm{~h})$ and $\mathrm{Ru}(\operatorname{cod})(\cot ) \quad(\operatorname{cod}=1,5$-cyclooctadiene; $\cot =1,3,5$-cyclooctatriene) $(\mathbf{1 6}, 16 \% ; \mathbf{1 7}, 1 \% ; 24 \mathrm{~h})$ were found to be active catalysts, but their activities were low compared with 3. In the case of the reaction of $\mathbf{1 5}$ with $\mathbf{2}$, $\mathrm{RhCl}\left(\mathrm{PPh}_{3}\right)_{3}(7)$, which was the active catalyst for the reaction of the ketone hydrazone 1 with olefins, did not serve as a catalyst. To find a more reactive hydrazone derivative, we further examined the present coupling reaction using several additional hydrazones. In the case of the reaction of a hydrazone having an $\mathrm{N}$-piperidyl group on the $s p^{2}$ nitrogen atom, the reactivity of the hydrazone was slightly increased (Table 2, run 5). However, when a sterically hindered piperidyl group, i.e., 2,6-dimethylpiperidyl group, was introduced on the $s p^{2}$ nitrogen, the yield was decreased considerably to $15 \%$ (Table 2 , run 6).

In summary, we report on a new procedure for chelationassisted $\mathrm{C}-\mathrm{H} /$ olefin coupling using transition metal-catalysts, which enables the site-selective alkylation of an aromatic hydrazone with olefins. Further studies to address the scope of this type of coupling reaction are now in progress.

\section{Acknowledgement}

This work was supported, in part, by a Grant-in-Aid for Scientific Research from Monbusho, Japan. F.K. thanks the Shorai Foundation for Science and Technology for financial support.
Table 2 The Transition Metal-catalyzed Reaction of Hydrazones with Olefins ${ }^{\mathrm{a}}$

\begin{tabular}{|c|c|c|c|c|c|c|c|c|c|c|}
\hline \multirow[t]{2}{*}{ run } & \multicolumn{4}{|c|}{ hydrazone } & olefin & cat. & time & \multicolumn{3}{|c|}{ yields $^{b}$} \\
\hline & & $\mathrm{R}^{1}$ & $\mathrm{R}^{2}$ & $\mathrm{NR}^{3}{ }_{2}$ & $Y$ & & & & & \\
\hline 1 & 9: & $\mathrm{H}$ & $\mathrm{Me}$ & $\mathrm{N}\left(\mathrm{CH}_{2}\right)_{5}$ & $\mathrm{Si}(\mathrm{OEt})_{3}$ & 7 & $8 \mathrm{~h}$ & $1027 \%$ & & $73 \%$ \\
\hline $2^{c}$ & 1: & $\mathrm{H}$ & $\mathrm{Me}$ & $\mathrm{NMe}_{2}$ & $\mathrm{H}$ & 7 & $24 \mathrm{~h}$ & $125 \%$ & & $38 \%$ \\
\hline 3 & 1: & $\mathrm{H}$ & $\mathrm{Me}$ & $\mathrm{NMe}_{2}$ & o-tolyl & 7 & $24 \mathrm{~h}$ & $1477 \%$ & & $0 \%$ \\
\hline $4^{\mathrm{d}, \mathrm{e}}$ & 15: & $\mathrm{Me}$ & $\mathrm{H}$ & $\mathrm{NMe}_{2}$ & $\mathrm{Si}(\mathrm{iOEt})_{3}$ & 3 & $48 \mathrm{~h}$ & $1630 \%$ & & - \\
\hline $5^{\mathrm{d}, \mathrm{f}}$ & 18: & $\mathrm{Me}$ & $\mathrm{H}$ & $\mathrm{N}\left(\mathrm{CH}_{2}\right)_{5}$ & $\mathrm{Si}(\mathrm{OEt})_{3}$ & 3 & $48 \mathrm{~h}$ & $1951 \%$ & & - \\
\hline $6^{d}$ & 21: & $\mathrm{Me}$ & $\mathrm{H}$ & & $\mathrm{Si}(\mathrm{OEt})_{3}$ & 3 & $48 \mathrm{~h}$ & $2215 \%$ & & - \\
\hline
\end{tabular}

${ }^{\text {a}}$ Reaction conditions: hydrazone ( $\left.2 \mathrm{mmol}\right)$, olefin $(10 \mathrm{mmol})$, catalyst $(0.12 \mathrm{mmol})$, mesitylene $3 \mathrm{~mL}$, reflux,

${ }^{\mathrm{b}} \mathrm{GC}$ yield.

${ }^{\mathrm{c}}$ The reaction with ethylene ( $\left.7 \mathrm{~atm}, 14 \mathrm{mmol}\right)$ was carried out in autoclave at $150{ }^{\circ} \mathrm{C}$ (oil bath temperature).

${ }^{\mathrm{d}}$ The reaction was carried out in toluene.

${ }^{\mathrm{e}}$ The corresponding dehydrogenated product $\mathbf{1 7}$ was also obtained in

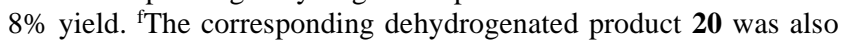
obtained in $7 \%$ yield.

\section{References and Notes}

(1) Kakiuchi, F.; Murai, S. Activation of C-H Bonds: Catalytic Reactions In Topics in Organometallic Chemistry, Murai, S., Ed.; Springer-Verlag: Berlin, 1999, Vol. 3, pp 47-79.

(2) (a) For examples, see: Murai, S.; Kakiuchi, F.; Sekine, S.; Tanaka, Y.; Kamatani, A.; Sonoda, M.; Chatani, N. Pure Appl. Chem. 1994, 66, 1527. (b) Kakiuchi, F.; Sekine, S.; Tanaka, Y.; Kamatani, A.; Sonoda, M.; Chatani, S.; Murai, S. Bull. Chem. Soc. Jpn. 1995, 68, 62. (c) Sonoda, M.; Kakiuchi, F.; Chatani, N.; Murai, S. Bull. Chem. Soc. Jpn. 1997, 70, 3117.

(3) Grigg, R.; Savic, V. Tetrahedron Lett. 1997, 38, 5737. Guari, Y.; Sabo-Etienne, S.; Chaudret, B. J. Am. Chem. Soc. 1998, 120, 4228. Busch, S.; Leitner, W. Chem. Commun. 1999, 2305. Harris, P. W. R.; Rickard, C. E. F.; Woodgate, P. D. J. Organomet. Chem. 2000, 601, 172. Gupta, S. K.; Weber, W. P. Macromolecules 2000, 33, 108.

(4) Sonoda, M.; Kakiuchi, F.; Kamatani, A.; Chatani, N.; Murai, S. Chem. Lett. 1996, 109.

(5) Trost, B. M.; Imi, K.; Davies, I. W. J. Am. Chem. Soc. 1995, $117,5371$.

(6) (a) Kakiuchi, F.; Yamauchi, M.; Chatani, N.; Murai, S. Chem. Lett. 1996, 111. (b) Kakiuchi, F.; Sato, T.; Tsujimoto, T.; Yamauchi, M.; Chatani, N.; Murai, S. Chem. Lett. 1998, 1053.

(7) Kakiuchi, F.; Sato, T.; Yamauchi, M.; Chatani, N.; Murai, S. Chem. Lett. 1999, 19.

(8) Kakiuchi, F.; Sonoda, M.; Tsujimoto, T.; Chatani, N.; Murai, S. Chem. Lett. 1999, 1083.

(9) Kakiuchi, F.; Sato, T.; Igi, K.; Chatani, N.; Murai, S. Chem. Lett., in press.

(10) For examples, see: Kakiuchi, F.; Yamada, A.; Chatani, N.; Murai, S. Bull. Chem. Soc. Jpn. 1998, 71, 285. Kakiuchi, F.; Le Gendre, P.; Yamada, A.; Ohtaki, H.; Murai, S. Tetrahedron: Asymmetry 2000, 11, 2647.

(11) Lim, Y.-G.; Kang, J.-B.; Kim, Y. H. J. Chem. Soc., Perkin Trans. 1 1996, 2201. 
(12) Matsubara, T.; Koga, N.; Musaev, D. G.; Morokuma, K. J. Am. Chem. Soc. 1998, 120, 12692. Matsubara, T.; Koga, N.; Musaev, D. G.; Morokuma, K. Organometallics 2000, 19, 2318.

(13) Said, S. B.; Skarzewski, J.; Mlochowski, J. Synthesis 1989, 223. Stankovic, S.; Espenson, J. H. Chem. Commun. 1998, 1579.

(14) Enders, D.; Schubert, H.; Nubling, C. Angew. Chem., Int. Ed. Engl. 1986, 25, 1109. Denmark, S. E.; Weber, T.; Piotrowski, D. W. J. Am. Chem. Soc. 1987, 109, 2224.

(15) General procedure for the reaction of aromatic hydrazones with olefins. An apparatus consisting of a $10 \mathrm{~mL}$ two-necked flask, a reflux condenser connected to a nitrogen line, a magnetic stirring bar, and an inlet-tube sealed with a rubber septum was flame-dried under a flow of nitrogen. After cooling to room temperature, the following reagents were placed in the flask: catalyst $(0.12 \mathrm{mmol}), 3 \mathrm{~mL}$ of toluene, hydrazone (2 mmol), olefin (10 mmol), and hexadecane (an internal standard for GC analysis). The resulting solution was then refluxed with stirring. The reaction was monitored by GC analysis. After heating for the appropriate reaction period, the solution was then concentrated in vacuo, and the residue purified by bulb-to-bulb distillation and/or silica gel column chromatography.

(16) 4 (anti isomer): ${ }^{1} \mathrm{H} \mathrm{NMR}\left(\mathrm{CDCl}_{3}, 270 \mathrm{MHz}\right) \delta 0.92-1.00$ (c, 2 $\left.\mathrm{H}, \mathrm{SiCH}_{2}\right), 1.22\left(\mathrm{t}, J=7.02 \mathrm{~Hz}, 9 \mathrm{H}, \mathrm{CH}_{2} \mathrm{CH}_{3}\right), 2.30(\mathrm{~s}, 3 \mathrm{H}$, $\left.\mathrm{CH}_{3}\right), 2.61\left(\mathrm{~s}, 6 \mathrm{H}, \mathrm{NCH}_{3}\right), 2.74-2.80\left(\mathrm{c}, 2 \mathrm{H}, \mathrm{CH}_{2}\right), 3.82$ (q, $\left.J=7.02 \mathrm{~Hz}, 6 \mathrm{H}, \mathrm{OCH}_{2}\right), 7.0-7.3(\mathrm{~m}, 4 \mathrm{H}, \mathrm{ArH}) ;{ }^{13} \mathrm{C} \mathrm{NMR}$ $\left(\mathrm{CDCl}_{3}, 67.5 \mathrm{MHz}\right) \delta 12.85,18.26,19.48,26.22,46.96,58.33$, 125.71, 127.51, 128.27, 128.95, 139.59, 141.76, 166.00; MS (\% relative intensity) $352\left(\mathrm{M}^{+}, 13\right), 163\left(\left[\mathrm{Si}(\mathrm{OEt})_{3}\right]^{+}, 100\right)$. HRMS: Calcd for $\mathrm{C}_{18} \mathrm{H}_{32} \mathrm{~N}_{2} \mathrm{O}_{3} \mathrm{Si}: 352.2182$. Found: 352.2198 . 4 (syn isomer): ${ }^{1} \mathrm{H}$ NMR $\left(\mathrm{CDCl}_{3}, 270 \mathrm{MHz}\right) \delta 0.92-0.99(\mathrm{c}, 2$ $\left.\mathrm{H}, \mathrm{SiCH}_{2}\right), 1.23$ (t, J=7.02 Hz, $\left.9 \mathrm{H}, \mathrm{CH}_{2} \mathrm{CH}_{3}\right), 2.20$ (s, $3 \mathrm{H}$, $\left.\mathrm{CH}_{3}\right), 2.35$ (s, $\left.6 \mathrm{H}, \mathrm{NCH}_{3}\right), 2.74-2.80\left(\mathrm{c}, 2 \mathrm{H}, \mathrm{CH}_{2}\right), 3.84$ (q, $\left.J=7.02 \mathrm{~Hz}, 6 \mathrm{H}, \mathrm{OCH}_{2}\right), 7.0-7.3(\mathrm{~m}, 4 \mathrm{H}, \mathrm{ArH}) ;{ }^{13} \mathrm{C} \mathrm{NMR}$ $\left(\mathrm{CDCl}_{3}, 67.5 \mathrm{MHz}\right) \delta 11.45,18.26,26.02,26.22,46.96,58.37$, $125.50,126.09,127.94,128.14,138.74,140.07,163.09$. 5: ${ }^{1} \mathrm{H}$ NMR $\left(\mathrm{CDCl}_{3}, 270 \mathrm{MHz}\right) \delta 1.22(\mathrm{t}, J=7.02 \mathrm{~Hz}, 9 \mathrm{H}$, $\left.\mathrm{CH}_{2} \mathrm{CH}_{3}\right), 2.19\left(\mathrm{~s}, 3 \mathrm{H}, \mathrm{CH}_{3}\right), 2.35\left(\mathrm{~s}, 6 \mathrm{H}, \mathrm{NCH}_{3}\right), 3.84$ (q, $\left.J=7.02 \mathrm{~Hz}, \mathrm{OCH}_{2}\right), 6.10(\mathrm{~d}, J=17.8 \mathrm{~Hz}, 1 \mathrm{H}, \mathrm{SiCH}=), 7.0$ $7.4(\mathrm{~m}, 4 \mathrm{H}, \mathrm{ArH}), 7.40(\mathrm{~d}, J=1 \mathrm{H}, \mathrm{CH}=)$.

6 (anti isomer): ${ }^{1} \mathrm{H} \mathrm{NMR}\left(\mathrm{CDCl}_{3}, 270 \mathrm{MHz}\right) \delta 0.84-1.08$ (c, 4 $\left.\mathrm{H}, \mathrm{SiCH}_{2}\right), 1.22\left(\mathrm{t}, J=7.02 \mathrm{~Hz}, 18 \mathrm{H}, \mathrm{CH}_{2} \mathrm{CH}_{3}\right), 2.26(\mathrm{~s}, 3 \mathrm{H}$, $\left.\mathrm{CH}_{3}\right), 2.60\left(\mathrm{~s}, 6 \mathrm{H}, \mathrm{NCH}_{3}\right), 2.57-2.63\left(\mathrm{c}, 4 \mathrm{H}, \mathrm{CH}_{2}\right), 3.81$ (q, $\left.J=7.02 \mathrm{~Hz}, 12 \mathrm{H}, \mathrm{OCH}_{2}\right), 7.0-7.3(\mathrm{~m}, 3 \mathrm{H}, \mathrm{ArH}) ;{ }^{13} \mathrm{C} \mathrm{NMR}$ $\left(\mathrm{CDCl}_{3}, 67.5 \mathrm{MHz}\right) \delta 12.67,18.28,19.97,25.99,46.90,58.33$, 125.32, 125.82, 139.48, 141.29, 166.65; MS (\% relative intensity) $542\left(\mathrm{M}^{+}, 20\right), 163\left(\left[\mathrm{Si}(\mathrm{OEt})_{3}\right]^{+}, 100\right)$. Anal Calcd for $\mathrm{C}_{26} \mathrm{H}_{50} \mathrm{~N}_{2} \mathrm{O}_{6} \mathrm{Si}_{2}:$ C, 57.53; H, 9.28; N, 5.16. Found: C, 57.48; $\mathrm{H}, 9.07 ; \mathrm{N}, 5.25 .6$ (syn isomer): ${ }^{1} \mathrm{H} \mathrm{NMR}\left(\mathrm{CDCl}_{3}, 270 \mathrm{MHz}\right)$ $\delta$ 0.84-1.08 (c, $\left.4 \mathrm{H}, \mathrm{SiCH}_{2}\right), 1.24(\mathrm{t}, J=7.02 \mathrm{~Hz}, 18 \mathrm{H}$, $\left.\mathrm{CH}_{2} \mathrm{CH}_{3}\right), 2.17\left(\mathrm{~s}, 3 \mathrm{H}, \mathrm{CH}_{3}\right), 2.35\left(\mathrm{~s}, 6 \mathrm{H}, \mathrm{NCH}_{3}\right), 2.57-2.63$ $\left(\mathrm{c}, 4 \mathrm{H}, \mathrm{CH}_{2}\right), 3.84\left(\mathrm{q}, J=7.02 \mathrm{~Hz}, 12 \mathrm{H}, \mathrm{OCH}_{2}\right), 7.0-7.3(\mathrm{~m}$, $3 \mathrm{H}, \mathrm{ArH}) ;{ }^{13} \mathrm{C} \mathrm{NMR}\left(\mathrm{CDCl}_{3}, 67.5 \mathrm{MHz}\right) \delta 11.70,18.28$, 25.86, 26.04, 46.60, 58.40, 128.00, 128.10, 137.65, 138.19, 161.81 .

10 (anti isomer): ${ }^{1} \mathrm{H}$ NMR $\left(\mathrm{CDCl}_{3}, 270 \mathrm{MHz}\right) \delta 0.88-1.04$ (c, $2 \mathrm{H}, \mathrm{SiCH}_{2}$ ), $1.23\left(\mathrm{t}, J=7.02 \mathrm{~Hz}, 9 \mathrm{H}, \mathrm{CH}_{2} \mathrm{CH}_{3}\right), 1.35$ (quintet, $J=5.67 \mathrm{~Hz}, 2 \mathrm{H}, \mathrm{CH}_{2}$ ); 1.73 (quintet, $J=5.67 \mathrm{~Hz}, 4 \mathrm{H}$, $\left.\mathrm{NCH}_{2} \mathrm{CH}_{2}\right), 2.30\left(\mathrm{~s}, 3 \mathrm{H}, \mathrm{CH}_{3}\right), 2.64-2.80\left(\mathrm{c}, 2 \mathrm{H}, \mathrm{ArCH}_{2}\right)$, $2.80\left(\mathrm{t}, J=5.67 \mathrm{~Hz}, 4 \mathrm{H}, \mathrm{NCH}_{2}\right), 3.82(\mathrm{q}, J=7.02 \mathrm{~Hz}, 6 \mathrm{H}$, $\left.\mathrm{OCH}_{2}\right), 7.0-7.3(\mathrm{~m}, 4 \mathrm{H}, \mathrm{ArH}) ;{ }^{13} \mathrm{C} \mathrm{NMR}\left(\mathrm{CDCl}_{3}, 67.5 \mathrm{MHz}\right)$ $\delta 12.89,18.26,19.61,25.25,25.39,26.06,55.90,58.31$, $125.68,127.55,128.16,128.93,139.69,141.80,165.97$; MS (\% relative intensity) $392\left(\mathrm{M}^{+}, 49\right), 163\left(\left[\mathrm{Si}(\mathrm{OEt})_{3}\right]^{+}, 100\right)$. Anal Calcd for $\mathrm{C}_{21} \mathrm{H}_{36} \mathrm{~N}_{2} \mathrm{O}_{3} \mathrm{Si}$ : C, 64.24; H, 9.24; N, 7.13.
Found: C, 64.26; H, 9.46; N, 6.83. 10 (syn isomer): ${ }^{1} \mathrm{H}$ NMR $\left(\mathrm{CDCl}_{3}, 270 \mathrm{MHz}\right) \delta 0.88-1.04\left(\mathrm{c}, 2 \mathrm{H}, \mathrm{SiCH}_{2}\right), 1.23(\mathrm{t}$, $J=7.02 \mathrm{~Hz}, 9 \mathrm{H}, \mathrm{CH}_{2} \mathrm{CH}_{3}$ ), 1.29 (quintet, $J=5.67 \mathrm{~Hz}, 2 \mathrm{H}$, $\mathrm{CH}_{2}$ ); 1.48 (quintet, $J=5.67 \mathrm{~Hz}, 4 \mathrm{H}, \mathrm{NCH}_{2} \mathrm{CH}_{2}$ ), 2.21 (s, 3 $\left.\mathrm{H}, \mathrm{CH}_{3}\right), 2.59\left(\mathrm{t}, J=5.67 \mathrm{~Hz}, 4 \mathrm{H}, \mathrm{NCH}_{2}\right), 2.64-2.80(\mathrm{c}, 2 \mathrm{H}$, $\left.\mathrm{ArCH}_{2}\right), 3.83\left(\mathrm{q}, J=7.02 \mathrm{~Hz}, 6 \mathrm{H}, \mathrm{OCH}_{2}\right), 7.0-7.3(\mathrm{~m}, 4 \mathrm{H}$, $\mathrm{ArH}) ;{ }^{13} \mathrm{C} \mathrm{NMR}\left(\mathrm{CDCl}_{3}, 67.5 \mathrm{MHz}\right) \delta 12.89,18.26,23.78$, 23.90, 26.06, 26.17, 55.71, 58.31, 125.21, 126.13, 127.64, 127.91, 138.72, 140.36, 163.68.

11 (anti isomer): ${ }^{1} \mathrm{H} \mathrm{NMR}\left(\mathrm{CDCl}_{3}, 270 \mathrm{MHz}\right) \delta 0.80-1.11$ (c, $\left.4 \mathrm{H}, \mathrm{SiCH}_{2}\right), 1.22\left(\mathrm{t}, J=7.02 \mathrm{~Hz}, 18 \mathrm{H}, \mathrm{CH}_{3}\right), 1.48$ (quint, $J=5.67 \mathrm{~Hz}, 2 \mathrm{H}, \mathrm{CH}_{2}$ ), 1.72 (quint, $J=5.67 \mathrm{~Hz}, 4 \mathrm{H}$, $\left.\mathrm{NCH}_{2} \mathrm{CH}_{2}\right), 2.27\left(\mathrm{~s}, 3 \mathrm{H}, \mathrm{CH}_{3}\right), 2.57-2.63\left(\mathrm{c}, 4 \mathrm{H}, \mathrm{ArCH}_{2}\right)$, $2.80\left(\mathrm{t}, J=5.67 \mathrm{~Hz}, 4 \mathrm{H}, \mathrm{NCH}_{2}\right), 3.82(\mathrm{q}, J=7.02 \mathrm{~Hz}, 12 \mathrm{H}$, $\left.\mathrm{OCH}_{2}\right), 7.0-7.3(\mathrm{~m}, 3 \mathrm{H}, \mathrm{ArH}) ;{ }^{13} \mathrm{C} \mathrm{NMR}\left(\mathrm{CDCl}_{3}, 67.5 \mathrm{MHz}\right)$ $\delta 13.19,18.74,20.52,24.40,25.88,26.42,56.28,58.78$, 126.27, 128.50, 138.74, 141.82, 167.12; MS (\% relative intensity) $582\left(\mathrm{M}^{+}, 100\right), 163\left(\left[\mathrm{Si}(\mathrm{OEt})_{3}\right]^{+}, 41\right)$. Anal Calcd for $\mathrm{C}_{29} \mathrm{H}_{54} \mathrm{~N}_{2} \mathrm{O}_{6} \mathrm{Si}_{2}$ : C, 59.75; H, 9.34; N, 4.81. Found: C, 59.92, $\mathrm{H}, 9.42 ; 4.90 .11$ (syn isomer): ${ }^{1} \mathrm{H} \mathrm{NMR}\left(\mathrm{CDCl}_{3}, 270 \mathrm{MHz}\right) \delta$ 0.80-1.11 (c, $4 \mathrm{H}, \mathrm{SiCH}_{2}$ ), 1.23 (quintet, $J=5.67 \mathrm{~Hz}, 2 \mathrm{H}$, $\left.\mathrm{CH}_{2}\right), 1.24\left(\mathrm{t}, J=7.02 \mathrm{~Hz}, 18 \mathrm{H}, \mathrm{CH}_{3}\right), 1.35$ (quintet, $J=5.67$ $\left.\mathrm{Hz}, 4 \mathrm{H}, \mathrm{NCH}_{2} \mathrm{CH}_{2}\right), 2.17$ (s, $\left.3 \mathrm{H}, \mathrm{CH}_{3}\right), 2.57-2.63(\mathrm{c}, 4 \mathrm{H}$, $\left.\mathrm{ArCH}_{2}\right), 2.66\left(\mathrm{t}, J=5.67 \mathrm{~Hz}, 4 \mathrm{H}, \mathrm{NCH}_{2}\right), 3.84(\mathrm{q}, J=7.02$ $\left.\mathrm{Hz}, 12 \mathrm{H}, \mathrm{OCH}_{2}\right), 7.0-7.3(\mathrm{~m}, 3 \mathrm{H}, \mathrm{ArH}) ;{ }^{13} \mathrm{C} \mathrm{NMR}\left(\mathrm{CDCl}_{3}\right.$, $67.5 \mathrm{MHz}) \delta 13.19,18.74,24.28,25.77,26.42,55.76,58.85$, $125.61,128.12,138.20,140.00,167.12$.

12: ${ }^{1} \mathrm{H}$ NMR $\left(\mathrm{CDCl}_{3}, 270 \mathrm{MHz}\right) \delta 1.25(\mathrm{t}, J=7.56 \mathrm{~Hz}, 6 \mathrm{H}$, $\left.\mathrm{CH}_{2} \mathrm{CH}_{3}\right), 2.15\left(\mathrm{~s}, 3 \mathrm{H}, \mathrm{CH}_{3}\right), 2.60\left(\mathrm{~s}, 6 \mathrm{H}, \mathrm{NCH}_{3}\right), 2.69$ (q, $\left.J=7.56 \mathrm{~Hz}, 4 \mathrm{H}, \mathrm{CH}_{2}\right), 7.0-7.3(\mathrm{~m}, 3 \mathrm{H}, \mathrm{ArH})$; $\mathrm{MS}$ (\% relative intensity) $190\left(\mathrm{M}^{+}, 14\right), 148\left(\left(\mathrm{M}-\mathrm{NMe}_{2}\right)^{+}, 48\right)$.

13 (anti isomer): ${ }^{1} \mathrm{H} \mathrm{NMR}\left(\mathrm{CDCl}_{3}, 270 \mathrm{MHz}\right) \delta 1.25$ (t, $\left.J=7.56 \mathrm{~Hz}, 6 \mathrm{H}, \mathrm{CH}_{2} \mathrm{CH}_{3}\right), 2.25\left(\mathrm{~s}, 3 \mathrm{H}, \mathrm{CH}_{3}\right), 2.52$ (q, $\left.J=7.56 \mathrm{~Hz}, 4 \mathrm{H}, \mathrm{CH}_{2}\right), 2.60\left(\mathrm{~s}, 6 \mathrm{H}, \mathrm{NCH}_{3}\right), 7.0-7.3(\mathrm{~m}, 3 \mathrm{H}$, ArH). 13 (syn isomer): ${ }^{1} \mathrm{H}$ NMR $\left(\mathrm{CDCl}_{3}, 270 \mathrm{MHz}\right) \delta 1.22(\mathrm{t}$, $\left.J=7.56 \mathrm{~Hz}, 6 \mathrm{H}, \mathrm{CH}_{2} \mathrm{CH}_{3}\right), 2.15\left(\mathrm{~s}, 3 \mathrm{H}, \mathrm{CH}_{3}\right), 2.35(\mathrm{~s}, 6 \mathrm{H}$, $\left.\mathrm{NCH}_{3}\right), 2.55\left(\mathrm{q}, J=7.56 \mathrm{~Hz}, 4 \mathrm{H}, \mathrm{CH}_{2}\right), 7.0-7.3(\mathrm{~m}, 3 \mathrm{H}, \mathrm{ArH})$. MS (\% relative intensity) $218\left(\mathrm{M}^{+}, 26\right), 174\left(\left[\mathrm{M}-\mathrm{NMe}_{2}\right]^{+}, 15\right)$. 14 (anti isomer): ${ }^{1} \mathrm{H} \mathrm{NMR}\left(\mathrm{CDCl}_{3}, 270 \mathrm{MHz}\right) \delta 2.23(\mathrm{~s}, 3 \mathrm{H}$, $\mathrm{CH}_{3}$ ), 2.30 (s, $\left.3 \mathrm{H}, \mathrm{ArCH}_{3}\right), 2.59$ (s, $6 \mathrm{H}, \mathrm{NCH}_{3}$ ), 2.88-2.96 (c, $\left.4 \mathrm{H}, \mathrm{CH}_{2}\right), 7.0-7.3$ (m, $\left.8 \mathrm{H}, \mathrm{ArH}\right) .{ }^{13} \mathrm{C} \mathrm{NMR}\left(\mathrm{CDCl}_{3}, 67.5\right.$ $\mathrm{MHz}$ (anti and syn isomers): $\delta 19.14,19.23,19.43,26.31$, $33.55,33.98,34.34,35.31,46.97,125.80,125.89,125.97$, $126.02,126.33,127.64,127.85,128.16,128.55,128.79$, $129.00,129.83,130.06,130.12,135.78,137.54,138.89$, 139.16, 139.93, 139.98, 162.26, 165.62; MS (\% relative intensity) $280\left(\mathrm{M}^{+}, 2\right), 221\left(\left[\mathrm{M}-\mathrm{NMe}_{3}\right]^{+}, 11\right)$. Anal Calcd for $\mathrm{C}_{19} \mathrm{H}_{24} \mathrm{~N}_{2}$ : C, 81.38; H, 8.63; N, 9.99. Found: C, 81.29; H, 8.59; N, 9.94. 14 (syn isomer): ${ }^{1} \mathrm{H}$ NMR $\left(\mathrm{CDCl}_{3}, 270 \mathrm{MHz}\right) \delta$ $2.14\left(\mathrm{~s}, 3 \mathrm{H}, \mathrm{CH}_{3}\right), 2.32$ (s, $\left.3 \mathrm{H}, \mathrm{ArCH}_{3}\right), 2.35\left(\mathrm{~s}, 6 \mathrm{H}, \mathrm{NCH}_{3}\right)$, 2.88-2.96 (c, $\left.4 \mathrm{H}, \mathrm{CH}_{2}\right), 7.0-7.3(\mathrm{~m}, 8 \mathrm{H}, \mathrm{ArH})$.

16: ${ }^{1} \mathrm{H} \mathrm{NMR}\left(\mathrm{CDCl}_{3}, 270 \mathrm{MHz}\right) \delta 0.94-1.00\left(\mathrm{c}, 2 \mathrm{H}, \mathrm{SiCH}_{2}\right)$, $1.23\left(\mathrm{t}, J=7.02 \mathrm{~Hz}, 9 \mathrm{H}, \mathrm{CH}_{3}\right), 2.39\left(\mathrm{~s}, 3 \mathrm{H}, \mathrm{ArCH}_{3}\right), 2.81-$ $2.88\left(\mathrm{c}, 2 \mathrm{H}, \mathrm{CH}_{2}\right), 2.95\left(\mathrm{~s}, 6 \mathrm{H}, \mathrm{NCH}_{3}\right), 3.83(\mathrm{q}, J=7.02 \mathrm{~Hz}$, $\left.6 \mathrm{H}, \mathrm{OCH}_{2}\right), 7.06(\mathrm{~m}, 3 \mathrm{H}, \mathrm{ArH}), 7.46(\mathrm{~s}, 1 \mathrm{H}, \mathrm{CH}=\mathrm{N}) ;{ }^{13} \mathrm{C}$ $\mathrm{NMR}\left(\mathrm{CDCl}_{3}, 67.5 \mathrm{MHz}\right) \delta 12.65,18.31,21.19,26.99,42.88$, 58.33, 126.79, 127.21, 128.36, 132.88, 133.05, 136.62, 143.29; MS (\% relative intensity) $352\left(\mathrm{M}^{+}, 9\right), 163$ $\left(\left[\mathrm{Si}(\mathrm{OEt})_{3}\right]^{+}, 100\right)$. Anal Calcd for $\mathrm{C}_{18} \mathrm{H}_{32} \mathrm{~N}_{2} \mathrm{O}_{3} \mathrm{Si}: \mathrm{C}, 61.32 ; \mathrm{H}$, 9.15; N, 7.94. Found: C, 61.43; H, 9.15; N, 7.72.

17: ${ }^{1} \mathrm{H}$ NMR $\left(\mathrm{CDCl}_{3}, 270 \mathrm{MHz}\right) \delta 1.10(\mathrm{t}, J=7.02 \mathrm{~Hz}, 9 \mathrm{H}$, $\left.\mathrm{CH}_{3}\right), 2,38\left(\mathrm{~s}, 3 \mathrm{H}, \mathrm{ArCH}_{3}\right), 2.97\left(\mathrm{~s}, 6 \mathrm{H}, \mathrm{NCH}_{3}\right), 3.88$ (q, $\left.J=7.02 \mathrm{~Hz}, 6 \mathrm{H}, \mathrm{CH}_{2}\right), 6.03(\mathrm{~d}, J=18.9 \mathrm{~Hz}, \mathrm{SiCH}=), 7.0-7.3$ $(\mathrm{m}, 3 \mathrm{H}, \mathrm{ArH}), 7.43(\mathrm{~s}, 1 \mathrm{H}, \mathrm{CH}=\mathrm{N}), 7.66(\mathrm{~d}, J=18.9 \mathrm{~Hz}$, $\mathrm{CH}=$ ). HRMS Calcd for $\mathrm{C}_{18} \mathrm{H}_{30} \mathrm{~N}_{2} \mathrm{O}_{3} \mathrm{Si}: 350.2026$. Found: 350.2030 . 
19: ${ }^{1} \mathrm{H} \mathrm{NMR}\left(\mathrm{CDCl}_{3}, 270 \mathrm{MHz}\right) \delta 0.93-1.00\left(\mathrm{c}, 2 \mathrm{H}, \mathrm{SiCH}_{2}\right)$, $1.23\left(\mathrm{t}, J=7.02 \mathrm{~Hz}, 9 \mathrm{H}, \mathrm{CH}_{3}\right), 1.55$ (quintet, $J=5.67 \mathrm{~Hz}, 2$ $\mathrm{H}, \mathrm{CH}_{2}$ ), 1.76 (quintet, $J=5.67 \mathrm{~Hz}, 4 \mathrm{H}, \mathrm{NCH}_{2} \mathrm{CH}_{2}$ ), 2.39 (s, $\left.3 \mathrm{H}, \mathrm{ArCH}_{3}\right), 2.80-2.87\left(\mathrm{c}, 2 \mathrm{H}, \mathrm{ArCH}_{2}\right), 3.15(\mathrm{t}, J=5.67 \mathrm{~Hz}$, $\left.4 \mathrm{H}, \mathrm{NCH}_{2}\right), 3.82\left(\mathrm{q}, J=7.02 \mathrm{~Hz}, 6 \mathrm{H}, \mathrm{OCH}_{2}\right), 7.0-7.3(\mathrm{~m}, 3$ $\mathrm{H}, \mathrm{ArH}), 7.78(\mathrm{~s}, 1 \mathrm{H}, \mathrm{CH}=\mathrm{N}) ;{ }^{13} \mathrm{C} \mathrm{NMR}\left(\mathrm{CDCl}_{3}, 67.5 \mathrm{MHz}\right)$ $\delta 12.71,18.28,21.13,24.23,25.09,26.92,52.24,58.29$, 126.76, 127.40, 128.32, 133.05, 134.90, 136.71, 143.41; MS (\% relative intensity) $392\left(\mathrm{M}^{+}, 9\right), 163\left(\left[\mathrm{Si}(\mathrm{OEt})_{3}\right]^{+}, 100\right)$. HRMS Calcd for $\mathrm{C}_{21} \mathrm{H}_{36} \mathrm{~N}_{2} \mathrm{O}_{3}$ Si: 392.2495. Found: 392.2483 . 22: ${ }^{1} \mathrm{H}$ NMR $\left(\mathrm{CDCl}_{3}, 270 \mathrm{MHz}\right) \delta 0.95-1.02\left(\mathrm{c}, 2 \mathrm{H}, \mathrm{SiCH}_{2}\right)$, $1.06\left(\mathrm{~d}, J=6.48 \mathrm{~Hz}, 6 \mathrm{H}, \mathrm{CHCH}_{3}\right), 1.23(\mathrm{t}, J=7.02 \mathrm{~Hz}, 9 \mathrm{H}$, $\left.\mathrm{CH}_{3}\right), 1.5-1.6\left(\mathrm{~m}, 3 \mathrm{H}, \mathrm{CH}_{2}\right), 1.7-1.8\left(\mathrm{~m}, 3 \mathrm{H}, \mathrm{CH}_{2}\right), 2.47(\mathrm{~s}, 3$ $\left.\mathrm{H}, \mathrm{ArCH}_{3}\right), 2.89-2.96$ (c, $\left.2 \mathrm{H}, \mathrm{ArCH}_{2}\right), 3.0-3.1(\mathrm{~m}, 2 \mathrm{H}, \mathrm{CH})$,
3.87 (q, $\left.J=7.02 \mathrm{~Hz}, 6 \mathrm{H}, \mathrm{OCH}_{2}\right), 7.0-7.3(\mathrm{~m}, 3 \mathrm{H}, \mathrm{ArH}), 8.43$ $(\mathrm{s}, 1 \mathrm{H}, \mathrm{CH}=\mathrm{N}) ;{ }^{13} \mathrm{C} \mathrm{NMR}\left(\mathrm{CDCl}_{3}, 67.5 \mathrm{MHz}\right) \delta 12.72$,

18.31, 20.88, 21.57, 21.76, 27.05, 32.83, 57.14, 58.33, 126.92, $128.55,128.61,131.52,137.68,144.38,153.57$; MS (\% relative intensity) $420\left(\mathrm{M}^{+}, 8\right), 163\left(\left[\mathrm{Si}(\mathrm{OEt})_{3}\right]^{+}, 100\right)$. HRMS Calcd for $\mathrm{C}_{23} \mathrm{H}_{40} \mathrm{~N}_{2} \mathrm{O}_{3} \mathrm{Si}$ : 420.2808. Found: 420.2807 .

(17) Thermal syn-anti isomerization in the hydrazone appeared to occur during the reaction.

(18) We previously mentioned the possibility of cleavage of the second $\mathrm{C}-\mathrm{H}$ bond without dissociation of the 1:1 addition product from the metal center. See, ref. $2 \mathrm{a}$.

Article Identifier:

1437-2096,E;2001,0,SI,0948,0951,ftx,en;Y02001ST.pdf 